\title{
A New Approach for Protocol Analysis on Design Activities Using Axiomatic Theory of Design Modeling
}

\author{
Shengji Yao and Yong Zeng ${ }^{*}$ \\ Concordia Institute for Information Systems Engineering \\ Concordia University \\ 1455 de Maisonneuve West, CB-410-16, Montreal, Quebec H3G 1M8, Canada \\ *E-mail: zeng@ciise.concordia.ca
}

\begin{abstract}
Although various design methodologies have been developed to help designers generate design concepts and design ideas, they must be applied by designers. Designers play an important and critical role in delivering a successful and innovative design. To understand how designers think in solving a problem during the design process will help us develop a new design methodology that can accommodate designer's performance. This paper proposes a new protocol analysis approach to studying designer's cognitive behaviour during the design process by using the axiomatic theory of design modeling. The conventional protocol analysis approach, which includes transcripts, segmentation, encoding and experiment results analysis, largely depends on the experience of the people who conduct the analysis. In this research, the concept of design state, derived from the axiomatic theory of design modeling, is used to guide the entire protocol analysis process. The consistency of analyzing subject's protocol is checked by three operators. This paper reports our preliminary study of protocol analysis on design activities. Indepth report of this research will be given in our future work.
\end{abstract}

\section{Introduction}

Design is a subject that has been studied for many years. A lot of design theories and methodologies have been developed to assist designers to conduct design activities [1-8]. While those design theories and methodologies provide some guidelines and procedures for designers, they leave a lot of flexibility and space for designers to perform. The design solutions are varied with different designers. An innovative design solution is often delivered by a creative designer. Dasgupta indicated in his paper [9], “...... design agent's freedom and capacity to use his knowledge contribute significantly to the design creativity." One promising approach to covering the discrepancies between traditional design methodologies and designer's freedom is to study designers' cognitive processes when they are working on a design problem. Our ultimate goal is to develop robust design tools to enhance the creativity and innovation of human designers in making design decisions. To reach this goal, we must understand designer's thinking and mental process and what factors affect a designer's decision.

In this research, a protocol analysis method is developed to understand what factors are important in stimulating designers to generate creative ideas, by studying designer's thinking process and problemsolving behavior. Protocol analysis is a major technique in current research for attempting to identify the role of designer's thinking and reasoning in the design process [10-16]. This method is to study subject's mental processes in the performance of tasks by recording their spontaneous thinking aloud and subsequently segmenting the running commentary into the discrete atomic mental operations that the subjects have used in the accomplishment of the tasks [17]. There are two major approaches for protocol analysis: retrospective report and concurrent verbal report. The approach of concurrent verbal report is that designer is instructed to think-aloud and talk-aloud concurrently while performing a design task. The approach of retrospective report has two stages. At the first stage, the designers are left alone to conduct their design tasks without any interference. Then at the second 
stage, the designers are asked to recall their design process and are reminded to answer some questions if they miss some information. Concurrent reports have their advantages in tracking designer's thinking behavior at any time in the design process. However talking aloud might influence the subject's perception and thinking process. Lloyd, et al. [18] argued that although concurrent verbal reports can reveal some aspects of design thinking, there are many types of design thinking that remain impervious to concurrent verbalization requiring different methodologies for analysis. Suwa, et al [19] employed retrospective report to reveal the perceptual interactions between a designer and his own sketches because they think the talking aloud concurrently may interfere with participant's perception during their sketching activities. In retrospective protocols, designers might recall their design process partially and selectively, but this effect can be reduced by showing the designers the video that recorded his/her design process and by asking them some questions as memory cues. In our research, the retrospective report is employed to record designer's activities. The designers are first asked to think to solve a design problem and draw sketches to illustrate their design process if necessary. Then in the interview session, the designer will be asked to recall the design process and answer some questions if they miss some information.

The rest of this paper is organized as follows. Section 2 introduces the axiomatic theory of design modeling. Section 3 illustrates the experiment set-up. The experiment analysis is explained in Section 4. Section 5 presents a summary and the future work of this research.

\section{Axiomatic Theory of Design Modeling}

Our protocol analysis method is developed based on axiomatic theory of design modeling [8]; hence, it is essential to briefly introduce this theory before we discuss our protocol analysis. The axiomatic theory of design modeling [8] provides a formal approach that allows for the development of design theories following logical steps based on mathematical concepts and axioms. The details of axiomatic theory of design modeling can be found in [8].

A key concept in the axiomatic theory of design modeling is the structure operation, which can be defined as follows:

Structure operation, denoted by $\oplus$, is defined by the union $\cup$ of an object $\mathrm{O}$ and the interaction $\otimes$ of the object with itself.

$$
\oplus \mathrm{O}=\mathrm{O} \cup(\mathrm{O} \otimes \mathrm{O})
$$

where $\oplus \mathrm{O}$ is the structure of object $\mathrm{O}$.
The structure operation provides the aggregation mechanism for representing the object evolution in the design process. Based on the structure operation, the concept of product system can be defined.

A product system is the structure of an object $(\Omega)$ including both a product $(\mathrm{S})$ and its environment $(\mathrm{E})$.

$$
\Omega=\mathrm{E} \cup \mathrm{S}, \forall \mathrm{E}, \mathrm{S}[\mathrm{E} \cap \mathrm{S}=\Phi]
$$

where $\Phi$ is the object that is included in any object.

The product system $(\oplus \Omega)$ can then be expanded as follows:

$$
\oplus \Omega=\oplus(\mathrm{E} \cup \mathrm{S})=(\oplus \mathrm{E}) \cup(\oplus \mathrm{S}) \cup(\mathrm{E} \otimes \mathrm{S}) \cup(\mathrm{S} \otimes \mathrm{E})
$$

where $\oplus \mathrm{E}$ and $\oplus \mathrm{S}$ are structures of the environment and the product, respectively; $\mathrm{E} \otimes \mathrm{S}$ and $\mathrm{S} \otimes \mathrm{E}$ are the interactions between the environment and the product. A product system can be illustrated in Figure 1.

$\mathrm{E} \otimes \mathrm{S}$

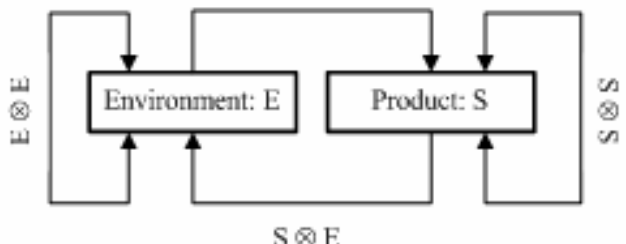

Figure 1. Product system

The design process can then be represented by design governing equation [20],

$$
\oplus \Omega_{\mathrm{i}+1}=\mathrm{D}_{\mathrm{i}}\left(\oplus \Omega_{\mathrm{i}}\right) \text {. }
$$

The design problem evolves along the evolution of the product. At each stage of this evolution process, the design problem is defined by its current product system $\oplus \Omega_{\mathrm{i}}$, which is called the state of the design. The components of product system $\oplus \Omega_{\mathrm{i}}$ keep on changing while conflicts exist in $\oplus \Omega_{\mathrm{i}}$. This evolution process of design can be illustrated as in Figure 2.

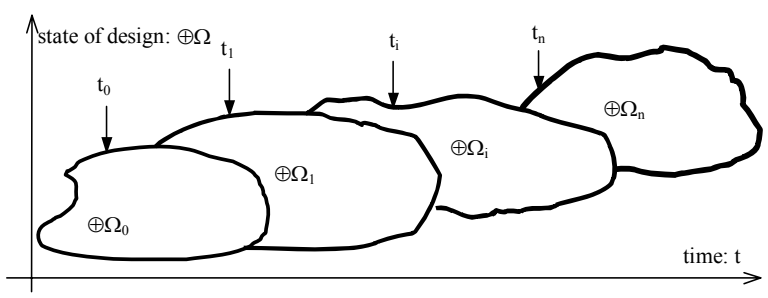

Figure 2. Evolution of the design process

The evolution process of design can be stated in the following theorem [20].

Theorem of Dynamic Structure of Design Problem. In the design process, design solutions to a design problem may change the original design problem, if the design solutions are different from their precedents, either by refinement or by alteration. 
This nature of the design problem was originally proposed by Zeng and Cheng as the recursive logic of design [21]. This logic indicates that design is a process recursively generating design solutions and the knowledge to evaluate the solutions. This result was confirmed by Roozenburg's research [22]. Later Maher, et al. [23] combined the gene concept with the recursive design process and pointed out that the problem space and the solution space co-evolve together, with interchange of information between the two spaces. Now design has been widely recognized as an evolution process with the change of the problem state and the solution state.

Considering the theorem of dynamic structure of design problem, we will identify each design state in the design process to analyze the subjects' experiment data. Based on the axiomatic theory of design modeling, we anticipate that in the design process, designers can figure out more environment components, deeper relationships between environment components and the product.

\section{Experiment}

\subsection{Design Problem}

Design problems should be selected based on their potential demands on different aspects of design skills. They should be practical, solvable, easily understood, suitable for designers with different levels. The design problem we have used in this paper is adapted from the research [24]. This design problem is to design a new litter-disposal system in a passenger compartment located in the trains of NS (Dutch Railways). The original description for this design problem can be found in [24]. Since the subjects in our experiment are not familiar with the train structure of Dutch Railways, we have modified the original design problem to make it more understandable to our subjects; the revised description of the design problem is shown as below.

\subsection{Subjects}

Subjects play an active role in our experiments. Seven graduate students from mechanical engineering, electrical engineering, and computer engineering participated in our experiment. We refer to the seven subjects as $\mathrm{S} 1$ to $\mathrm{S} 7$. We considered all the subjects as experienced designers since they have had various design experience from 5 to 10 years. All of the seven subjects have been trained in one or two design methodologies. In this research, we will use the design process of the subject $\mathrm{S} 1$ as an example to illustrate our protocol analysis method.
Design problem: to design a litter-disposal system for the passenger compartment. This system should be convenient for the passengers to deposit garbage and meanwhile it is easy for the cleaners to collect the garbage. The structure of the passenger compartment is as follows.

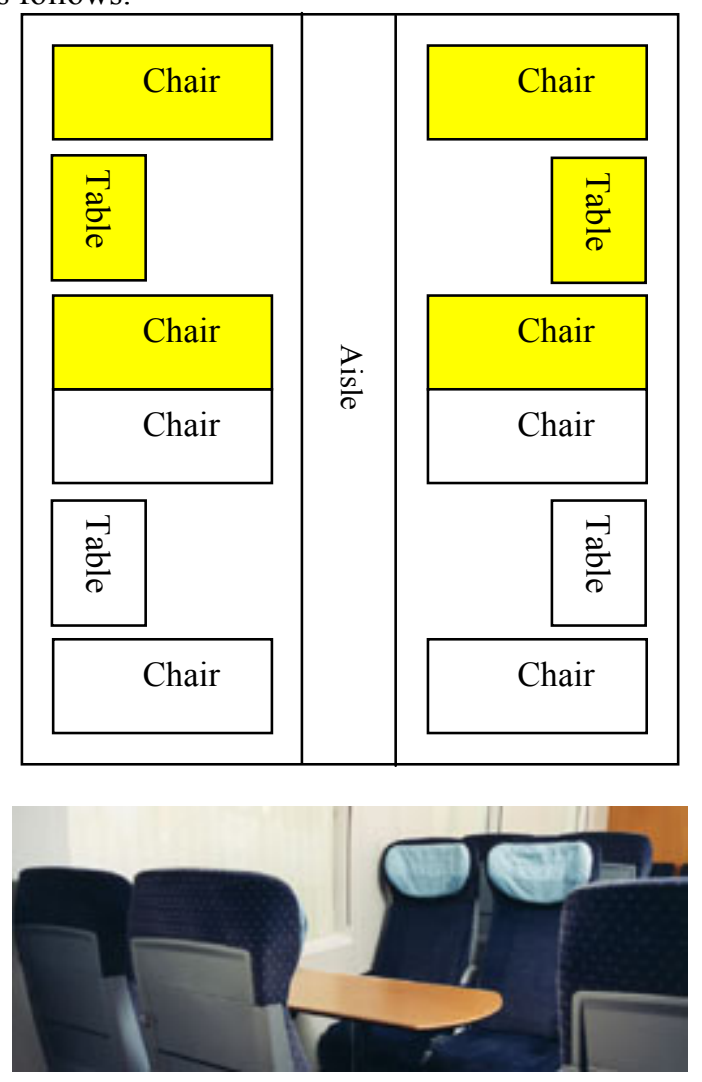

You are asked to generate a design concept for the design problem above. You need to write down all your ideas, concepts and draw the sketches to illustrate your solutions in your design process.

\subsection{Procedure}

Our experiment has two stages: design session and retrospection session. Design session refers to designer's problem-solving process and retrospection session refers to designer's retrospective process. In the first stage, subjects stay in a quiet room to solve the problem without any interference. If they have any question about the problem or need any further information, they can ask the experimenter. In this experiment, we set up three webcams to record the whole design process. One webcam is set up for general recording of the whole picture of the subject and his or her actions. The second webcam is set up for tracking the subject's subtle expression by recording his/her facial expression. The third webcam is set up to record the subject's gestures during the 
design process. The three webcams can record the audio and video information. The experiment set-up is shown in Figure 3.

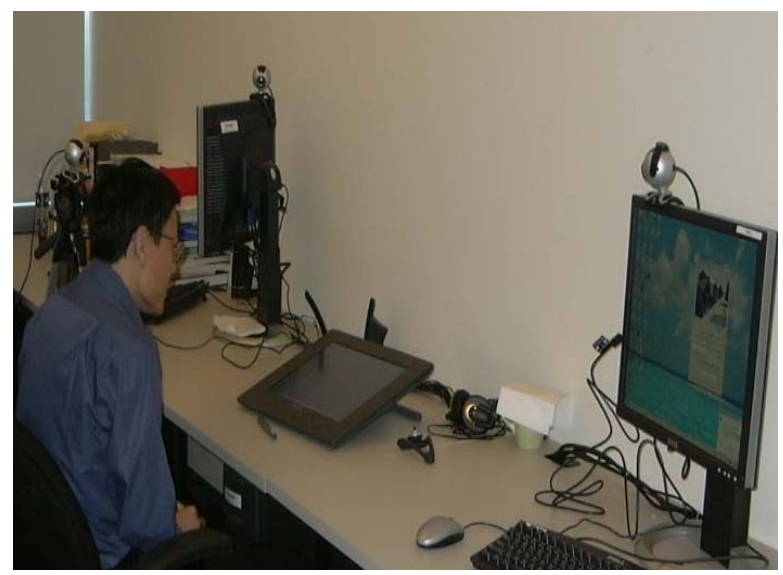

Figure 3. Experiment Set-up

Subjects are required to draw and write anything they want to solve the design problem on a WACOM tablet screen using the tablet pen with the help of the software "Procreate Painter Classic". This software is a kind of tool to support designers to draw or write anything on the screen. Before subjects begin to solve the problem, they are trained for 5-10 minutes to use the tablet pen and the tablet screen to draw sketches in order to feel as if they were using pen and paper. The activities done on the tablet screen by subjects are recorded by the software "My Screen Recorder". This software can record anything that subjects draw or write on the screen of the computer. Subjects' actions and the screen activities are monitored by the experimenter in the design process. When subjects finish solving the problem, the experimenter will understand designer's general actions and intentions.

In the second stage, we need to determine the subject's motivation towards the test situation and their own design. The subject is asked to recall and report what he/she was thinking at each step of his/her design process by watching the videos of his/her actions and the videos of his/her screen activities. If the subject misses some information, he/she will be reminded or asked to clarify his/her problem-solving behaviors. Meanwhile, the three webcams are used to record the whole retrospective process from different perspectives. Then the protocol data will be organized from the video and audio media and screen recording video for further analysis.

\section{Experiment Analysis}

After we conduct the experiment, we need to collect and organize the data from the experiment. First we need to transcribe what subjects spoke in the retrospective process from the videos. Then the transcribed data will be segmented and encoded for further analysis. The consistency and the accuracy of the protocol analysis need to be checked to ensure that the experiment analysis is more objective and more robust. The design solutions are evaluated based on the analysis using the axiomatic theory of design modeling. Then the results will be further conducted for statistical analysis.

\subsection{Transcription}

The first step of the experiment analysis is to transcribe all the words spoken by subjects during the retrospective stage into text documents. The text documents are taken as the initial transcripts. Then the initial transcripts are checked by a second operator to ensure the accuracy of the initial transcript. The text documents originally transcribed from subjects usually contain some vague and inconsistent information. Moreover, subjects may not give enough information to explain their thinking process just by showing their intentions. We also need to add some annotations to explain their non-verbal intentions to make the transcript more consistent. So a third operator is needed to filter the unnecessary information and add some annotations into the transcripts. If there are any discrepancies of the transcripts between the three operators, they will discuss to get the final agreement. Then we will get the formalized transcripts. A part of the formalized transcript of the subject $\mathrm{S} 1$ is shown below.

"First, I got your design problem. The first point of the design problem is that I want to make clear what the thing to be designed is. We made clear that we need to design a garbage bin. Then I think what the environment for this garbage bin is. In which place should it be put? Like this coach car or sleeping car? Then I consider the structure of the coach car. Now I consider the convenience for the cleaner to pick up the garbage bin. Cleaners walk along the aisle. Then I think to put the garbage bin under the table. It will affect the movement of the passenger's legs if the garbage bin is put under the table. So the only place is under the seats. Put here (under the seats). These are seats, tables (point to the sketches on the screen). This place (under the seats) is not convenient for picking up. Here, I feel it is not good. So, I consider putting along the side of the seats close to the aisle."

\subsection{Segmentation and encoding}

After we get the formalized transcripts, we need to divide the transcript of each subject into segments and 
then the segments are encoded using our coding scheme.

In this research, the transcript is divided into separate design states. A design state represents a single intention of the designer. From Section 2, we know that a design state is defined by the structure of environment components, the structure of product and the relationship between environment components and product. So in segmentation, a design state can be identified as any changes of environment components, any changes of product descriptions, or any change of the relationship between environment and product. An example of segmentation is given below by segmenting the above example of the formalized transcript.

Design State 1: "First, I got your design problem. The first point of the design problem is that I want to make clear what the thing to be designed is. We made clear that we need to design a garbage bin. Then I think what the environment for this garbage bin is. In which place should it be put? Like this coach car or sleeping car? Then I consider the structure of the coach car."

Design State 2: "Now I consider the convenience for the cleaner to pick up the garbage bin. Cleaners walk along the aisle. Then I think to put the garbage bin under the table. It will affect the movement of the passenger's legs if the garbage bin is put under the table."

Design State 3: "So the only place is under the seats. Put here (under the seats). These are seats, tables (point to the sketches on the screen). This place (under the seats) is not convenient for picking up. Here, I feel it is not good. So, I consider putting along the side of the seats close to the aisle."

Encoding is the most important part to analyze subject's protocol. Existing protocol studies in devising coding scheme first divide subjects' protocols into various design actions and encode design actions into some specific categories and subcategories. However those coding schemes are developed according to the specific design problem. If the design problem is changed, the coding scheme will also be changed. Since our encoding scheme is developed based on the axiomatic theory of design modeling, which implies the logic of design, it will enable us to systematically code designer's actions. Design process proceeds with the evolution of design states. So we will take each design state as the basic encoding unit. Then a design state will be decomposed into environment components, product, and the relationships between them. A design state (DS) is represented as $\oplus \Omega_{i}$. The structure of the product and the environment are represented as $\oplus \mathrm{S}_{\mathrm{i}}$ and $\oplus \mathrm{E}_{\mathrm{i}}$, respectively. An environment may contain several environment components $\mathrm{E}_{\mathrm{i}}=\sum_{\mathrm{j}} \mathrm{e}_{\mathrm{j}}$. Some environment components could be modified in different design states; therefore, the modified environment components will be encoded as $\mathrm{m}\left(\mathrm{e}_{\mathrm{i}}\right)$. The relationship between the environment and the product is encoded as $B_{i}=\left(E_{i} \otimes S_{i}\right) \cup\left(S_{i} \otimes E_{i}\right)$. If the relationships or the environment components are modified in different states, they will be encoded as $\mathrm{m}\left(\mathrm{B}_{\mathrm{i}}\right)$ and $\mathrm{m}\left(\mathrm{e}_{\mathrm{j}}\right)$, respectively. An example of the encoding is explained below. They are summarized in Table 1.

$$
\begin{aligned}
& \text { Design State 1: } \oplus \Omega_{1}=\oplus\left(\mathrm{E}_{1} \cup \mathrm{S}_{1}\right) \\
& E_{1}=e_{1}=\text { coach car } \\
& B_{1}=\text { where the garbage bin should be put in the } \\
& \text { coach car } \\
& S_{1}=\text { garbage bin }
\end{aligned}
$$$$
\text { Design State 2: } \oplus \Omega_{2}=\oplus\left(\mathrm{E}_{2} \cup \mathrm{S}_{2}\right)
$$$$
e_{2}=\text { cleaners, } e_{3}=\text { table }
$$$$
E_{2}=e_{1} \cup e_{2} \cup e_{3}
$$$$
B_{2}=\text { the convenience for the cleaner to pick up the }
$$$$
\text { garbage bin }
$$$$
S_{2}=\text { garbage bin is put under the table }
$$

Design State 3: $\oplus \Omega_{3}=\oplus\left(\mathrm{E}_{3} \cup \mathrm{S}_{3}\right)$

$e_{4}=$ passenger, $e_{5}=$ seats

$E_{3}=e_{1} \cup e_{2} \cup e_{3} \cup e_{4} \cup e_{5}$

$B_{3}$ : affect the leg of the passenger when put under the table and not convenient for picking up right under the seats.

$S_{3}=$ garbage bin is put along the side of the seats close to the aisle.

\subsection{Consistency}

Since the experiment analysis including transcription, segmentation, and encoding was conducted by people and it might contain subjectiveness, we need to assess the correctness and the consistency of the protocol analysis. In general, the consistency can be assessed in two ways. One way is to deal with the protocol data by the same person for several times and evaluate them repeatedly, like the Delphi method [25]. The other way is to deal with the protocol data in parallel by several people and then coordinate their results. In our study, the second 
method will be used to evaluate the experiment analysis. There are three operators to conduct the experiment analysis in our research. The first operator conducts the experiment analysis to get the initial transcript, segmentation and encoding. Then the second operator will check the results of the first operator and mark the results if he or she has a different opinion. Finally the third operator will coordinate the results of the two operators and make the final agreement for the experiment analysis. In this way, the consistency of our protocol analysis approach is maintained.

Table 1. An example of encoding

\begin{tabular}{|c|c|c|c|}
\hline No & $\begin{array}{c}E_{i}: \\
\text { environment }\end{array}$ & $\begin{array}{c}S_{i}: \\
\text { product }\end{array}$ & $\begin{array}{c}\qquad B_{i}: \\
\text { relationship }\end{array}$ \\
\hline DS1 & $\begin{array}{c}E_{1}=e_{1} \\
e_{1}=\text { coach car }\end{array}$ & $\begin{array}{l}\text { garbage } \\
\text { bin }\end{array}$ & $\begin{array}{l}\text { where the } \\
\text { garbage bin } \\
\text { should be put } \\
\text { in the coach } \\
\text { car? }\end{array}$ \\
\hline DS2 & $\begin{array}{c}E_{2}=e_{1} \\
\cup e_{2} \cup e_{3} \\
e_{2}=\text { cleaners } \\
e_{3}=\text { table }\end{array}$ & $\begin{array}{l}\text { garbage } \\
\text { bin is put } \\
\text { under the } \\
\text { table }\end{array}$ & $\begin{array}{l}\text { the } \\
\text { convenience } \\
\text { for the cleaner } \\
\text { to pick up the } \\
\text { garbage bin }\end{array}$ \\
\hline DS3 & $\begin{array}{c}E_{3}=e_{1} \cup e_{2} \\
\cup e_{3} \cup e_{4} \cup e_{5} \\
e_{4}=\text { passenger } \\
e_{5}=\text { seats }\end{array}$ & $\begin{array}{l}\text { garbage } \\
\text { bin is put } \\
\text { along the } \\
\text { side of the } \\
\text { seats close } \\
\text { to the aisle. }\end{array}$ & $\begin{array}{l}\text { affect the leg } \\
\text { of the } \\
\text { passenger } \\
\text { when put under } \\
\text { the table and } \\
\text { not convenient } \\
\text { for picking up } \\
\text { right under the } \\
\text { seats. }\end{array}$ \\
\hline
\end{tabular}

\subsection{Measurement of design solution}

The design solutions generated by the subjects need to be evaluated for comparisons. Previous research often used experts to set up some criteria to evaluate the design solutions. To avoid the subjectiveness, our study uses environment-based design [26] to analyze the design problem. All possible environment components and relations between environments and product will be listed. The potential conflicts in the design requirements will be identified. Then the design solutions will be measured based on the full analysis of the design problem using environmentbased design. The design solutions will be ranked according to how they match the analysis of the environment-based design. On the other hand, the design solutions will be measured according to the novelty and the value, which are the characteristics of creativity. Three assessors will evaluate the design solutions separately. The average measurements from the three assessors will be used as the final results.

\subsection{Statistical analysis}

We assume that the creative designer in his/her design process will consider more environment components, relationships and there are more transitions of evaluating environments between different design states. This assumption will be validated by the statistical analysis. The statistical analysis is based on some quantitative values and will be conducted from the design processes of the seven subjects. The following quantitative values will be summarized from the experiment results.

- Number of environment components in each state

- Number of environment-product relationships

- Number of repeated environment-product relation

- Number of concepts generated

- Time spent in the design process and in each design state

- Time spent in delivering a key concept

- Number of transitions/transition rate of evaluating the environment, the product and their relationships

- Number of conflicts.

This analysis is still on-going. The results will be reported in the future.

\section{Conclusions and Future Work}

This paper presented a protocol analysis approach for studying design activities. The axiomatic theory of design modeling is introduced as the basis of the protocol analysis approach. The experiment set-up is illustrated. We also investigated the experiment analysis including transcription, segmentation and encoding, consistency, the measurement of design solutions and the statistical analysis to be conducted. This paper is a preliminary study of protocol analysis on design activities; future work needs to be done to measure and compare the experiment results of different subjects. The comparisons between different designers will be checked to get observations and suggestions for guiding designers to make creative design decisions.

\section{Acknowledgement}

This work is partially supported by NSERC (Grant number RGPIN 298255). We would like to thank Honglin Yang and Imran Ur Rehman who assisted with data collection and analysis for this research. We 
also would like to express our thanks to all subjects of the experiment for their participation.

\section{References}

[1] Altshuller, G.S., Creativity as an Exact Science: the Theory of the Solutions of Inventive Problems. Studies in Cyberbetics, ed. F.H. George. 1984: Gordon and Breach Science Publishers.

[2] Braha, D. and O. Maimon, A Mathematical Theory of Design. 1998: Kluwer Academic Publishers.

[3] Ertas, A., M.M. Tanik, and T.T. Maxwell, "Transdisciplinary engineering education and research model". Transaction of the SDPS: Journal of

Integrated Design \& Process Science, 2000. 5(4): p. 121.

[4] Hubka, V. and W. Eder, Design Science. 1996: Springer. 231.

[5] Maxwell, T.T., A. Ertas, and M.M. Tanik, "Harnessing complexity in design". Transaction of SDPS: Journal of Integrated Design and Process Science, 2002. 6(3): p. 63-74.

[6] Pahl, G. and W. Beitz, Engineering Design: A Systematic Approach. 1988: Spinger-Verlag. [7] Suh, N., The Principles of Design. 1990: Oxford University Press.

[8] Zeng, Y., "Axiomatic theory of design modeling". Transaction of SDPS: Journal of Integrated Design and Process Science, 2002. 6(3): p. 1-28.

[9] Dasgupta, Creativity in Innovation and Design. 1994.

[10] Jin, Y. and P. Chusilp, "Study of mental iteration in different design situations". Design Studies, 2006. 27(1): p. 25-55.

[11] Atman, C.J., M.E. Cardella, J. Turns, and R. Adams, "Comparing freshman and senior engineering design processes: an in-depth follow-up study". Design Studies, 2005. 26(4): p. 325-357.

[12] Atman, C.J., J.R. Chimka, K.M. Bursic, and H.L. Nachtmann, "A comparison of freshman and senior engineering design processes". Design Studies, 1999. 20(2): p. 131-152.

[13] Suwa, M., T. Purcell, and J. Gero, "Macroscopic analysis of design processes based on a scheme for coding designers' cognitive actions". Design Studies, 1998. 19(4): p. 455-483.

[14] Gero, J.S. and T. Mc Neill, "An approach to the analysis of design protocols". Design Studies, 1998. 19(1): p. 21-61.

[15] Akin, O. and C. Akin, "On the process of creativity in puzzles, inventions, and designs". Automation in Construction, 1998. 7: p. 123-138. [16] Cross, N., "Descriptive models of creative design: application to an example". Design Studies, 1997. 18(4): p. 427-440. [17]http://www.informatics.susx.ac.uk/books/compute rs-and-thought/gloss/node1.html.

[18] Lloyd, P., B. Lawson, and P. Scott, "Can concurrent verbalization reveal design cognition?" Design Studies, 1995. 16(2): p. 237-259.

[19] Suwa, M. and B. Tversky, "What do architects and students perceive in their design sketches? A protocol analysis". Design Studies, 1997. 18(4): p. 385-403.

[20] Zeng, Y., "Environment-based formulation of design problem". Transaction of SDPS: Journal of Integrated Design and Process Science, 2004. 8(4): p. 45-63.

[21] Zeng, Y. and G. Cheng, "On the logic of design". Design Studies, 1991. 12(3): p. 137-141.

[22] Roozenburg, N.F.M., On the logic of innovative design, in Research in Design Thinking, N. Cross, K. Dorst, and N.F.M. Roozenburg, Editors. 1992, Delft University Press. p. 127-138.

[23] Maher, M.L. and H.-H. Tang, "Co-evolution as a computational and cognitive model of design".

Research in Engineering Design, 2003. 14: p. 47-63. [24] Dorst, K. and N. Cross, "Creativity in the design process: co-evolution of problem-solution". Design

Studies, 2001. 22(5): p. 425-437.

[25] Linsone, H.A. and M. Turoff, The Delphi Method Techniques and Applications. 1975, Addison-Wesley, Reading, MA.

[26] Zeng, Y., B.Q. Yan, B. Chen, and S.J. Yao. A

Theoretical and Experimental Study on Design

Creativity. in The Inaugural CDEN Design

Conference. 2004. Montreal, Canada. 